\title{
EXPONENTIAL STABILITY OF THE KIRCHHOFF PLATE WITH THERMAL OR VISCOELASTIC DAMPING
}

\author{
BY \\ ZHUANGYI LIU (Department of Mathematics and Statistics, University of Minnesota, Duluth, MN) \\ AND \\ SONGMU ZHENG (Department of Mathematics, Fudan University, Shanghai 200433, China)
}

\begin{abstract}
The exponential stability of the semigroup associated with the Kirchhoff plate with thermal or viscoelastic damping and various boundary conditions is proved. This improves the corresponding results by Lagnese by showing that the semigroup is still exponentially stable even without feedback control on the boundary. The proof is essentially based on PDE techniques and the method is remarkable in the sense that it also throws light on applications to other higher-dimensional problems.
\end{abstract}

1. Introduction. The purpose of this paper is to show that the semigroups associated with linear thermoelastic plates and linear viscoelastic plates of the Kirchhoff type with various boundary conditions are exponentially stable, which further leads to the exponential decay of energy of these plates.

Suppose a thin plate of the Kirchhoff type occupies a bounded region $\Omega \in \mathbf{R}^{2}$ with smooth boundary $\Gamma=\Gamma_{0} \cup \Gamma_{1} \cup \Gamma_{2}$. The plate is rigidly clamped along $\Gamma_{0}$, simply supported along $\Gamma_{1}$, and free along $\Gamma_{2}$. In addition, we assume that

$$
\bar{\Gamma}_{0} \cap \bar{\Gamma}_{1} \cap \bar{\Gamma}_{2}=\varnothing
$$

If thermal damping is considered, then the vertical deflection $w$ of the plate and the temperature $\theta$ satisfy the following partial differential equations (see $[\mathrm{L}]$ ):

$$
\begin{aligned}
w^{\prime \prime}-\gamma \Delta w^{\prime \prime}+\Delta^{2} w+\alpha \Delta \theta & =0, \\
\beta \theta^{\prime}-\eta \Delta \theta+\sigma \theta-\alpha \Delta w^{\prime} & =0,
\end{aligned}
$$

with $\alpha, \beta, \eta, \sigma>0, \gamma \geq 0$ being constants, and the prime being time derivative. Various boundary conditions could be imposed on $\theta$ depending on what is assumed about the

Received December 15, 1994.

1991 Mathematics Subject Classification. Primary 35B40, 73H10, 73K10, 73B30.

Key words and phrases. The Kirchhoff plate, thermal and viscoelastic damping, exponential stability, semigroup.

The second author was supported by NSFC 19331040 . 
temperature dynamics at the edge of the plate. We assume that the temperature is subject to the Newton law of cooling. Therefore, we have

$$
\begin{gathered}
w=\frac{\partial w}{\partial \nu}=0 \quad \text { on } \Gamma_{0}, \quad t>0 \\
w=\Delta w+(1-\mu) B_{1} w+\alpha \theta=0 \quad \text { on } \Gamma_{1}, \quad t>0 \\
\left\{\begin{array}{c}
\Delta w+(1-\mu) B_{1} w+\alpha \theta=0 \\
\frac{\partial \Delta w}{\partial \nu}+(1-\mu) \frac{\partial B_{2} w}{\partial \tau}-\gamma \frac{\partial w^{\prime \prime}}{\partial \nu}+\alpha \frac{\partial \theta}{\partial \nu}=0
\end{array} \text { on } \Gamma_{2}, \quad t>0\right. \\
\frac{\partial \theta}{\partial \nu}=-\lambda \theta \text { on } \Gamma, \quad t>0
\end{gathered}
$$

where $\nu=\left(\nu_{1}, \nu_{2}\right)$ is the unit outward normal to $\Gamma, \tau=\left\{-\nu_{2}, \nu_{1}\right\}$ is the unit tangent vector, $\lambda$ is a constant $(\lambda=0$ corresponds to the insulated temperature boundary condition), $\mu\left(\frac{1}{2}>\mu>0\right)$ is the Poisson ratio and

$$
\left\{\begin{array}{l}
B_{1} w=2 \nu_{1} \nu_{2} \frac{\partial^{2} w}{\partial x \partial y}-\nu_{1}^{2} \frac{\partial^{2} w}{\partial y^{2}}-\nu_{2}^{2} \frac{\partial^{2} w}{\partial x^{2}} \\
B_{2} w=\left(\nu_{1}^{2}-\nu_{2}^{2}\right) \frac{\partial^{2} w}{\partial x \partial y}+\nu_{1} \nu_{2}\left(\frac{\partial^{2} w}{\partial y^{2}}-\frac{\partial^{2} w}{\partial x^{2}}\right)
\end{array}\right.
$$

The initial state of the plate is

$$
w(0)=w^{0}(x, y), \quad w^{\prime}(0)=w^{1}(x, y), \quad \theta(0)=\theta^{0}(x, y)
$$

with $w^{0}, w^{1}, \theta^{0}$ being given functions.

The energy of the thermoelastic plate is defined by

$$
E(t)=\frac{1}{2}\left\{a(w(t))+\left\|w^{\prime}(t)\right\|^{2}+\gamma\left\|\nabla w^{\prime}(t)\right\|^{2}+\beta\|\theta\|^{2}\right\}
$$

where

$$
a(w(t))=\int_{\Omega}\left\{\left(\frac{\partial^{2} w}{\partial x^{2}}\right)^{2}+\left(\frac{\partial^{2} w}{\partial y^{2}}\right)^{2}+2 \mu \frac{\partial^{2} w}{\partial x^{2}} \frac{\partial^{2} w}{\partial y^{2}}+2(1-\mu)\left(\frac{\partial^{2} w}{\partial x \partial y}\right)^{2}\right\} d \Omega
$$

When viscoelastic damping is considered, the vertical deflection of the plate satisfies

$$
w^{\prime \prime}(t)-\gamma \Delta w^{\prime \prime}(t)+\Delta^{2} w(t)+\Delta^{2} \int_{0}^{\infty} g^{\prime}(s) w(t-s) d s=0
$$

with $\gamma \geq 0, g(0)=1$ (see $[\mathrm{L}])$. The corresponding boundary conditions are

$$
\begin{gathered}
w=\frac{\partial w}{\partial \nu}=0, \quad \text { on } \Gamma_{0}, \quad t>0 \\
w=\mathcal{B}_{1}\left(w(t)+\int_{0}^{\infty} g^{\prime}(s) w(t-s) d s\right)=0, \quad \text { on } \Gamma_{1}, \quad t>0
\end{gathered}
$$


and

$$
\left\{\begin{array}{l}
\mathcal{B}_{1}\left(w(t)+\int_{0}^{\infty} g^{\prime}(s) w(t-s) d s\right)=0 \\
\mathcal{B}_{2}\left(w(t)+\int_{0}^{\infty} g^{\prime}(s) w(t-s) d s\right)-\gamma \frac{\partial w^{\prime \prime}}{\partial \nu}=0
\end{array} \text { on } \Gamma_{2}, \quad t>0\right.
$$

where

$$
\mathcal{B}_{1} w=\Delta w+(1-\mu) B_{1} w, \quad \mathcal{B}_{2} w=\frac{\partial \Delta w}{\partial \nu}+(1-\mu) \frac{\partial B_{2} w}{\partial \tau}
$$

with the operators $B_{1}, B_{2}$ being the same as above and $\tau=\left\{-\nu_{2}, \nu_{1}\right\}$.

The initial state of the plate is

$$
w(0+)=w^{0}, \quad w^{\prime}(0+)=w^{1}, \quad w(-s)=w_{h}(s) \text { for } 0<s<\infty
$$

with $w^{0}, w^{1}, w_{h}$ being given functions.

We shall assume that the relaxation function $g(s)$ satisfies the following conditions: (g1) $g \in C^{2}(0, \infty) \cap C[0, \infty)$,

(g2) $g(t)>0, g^{\prime}(t)<0, g^{\prime \prime}(t)>0$, for $t>0$,

(g3) $g(\infty)=g_{\infty}>0$,

(g4) $g^{\prime \prime}(t)+k g^{\prime}(t) \geq 0$ for some $k>0$.

Condition (g4) implies that $g^{\prime}(t)$ decays exponentially, and (g3) means that the material behaves like an elastic solid at $t=\infty$.

The total energy corresponding to (1.12) is defined by

$$
\begin{array}{r}
E(t)=\frac{1}{2} \int_{\Omega}\left(g_{\infty} a(w(t))+\left[w^{\prime}(t)\right]^{2}+\gamma\left[\nabla w^{\prime}(t)\right]^{2}\right) d \Omega \\
-\frac{1}{2} \int_{0}^{\infty} \int_{\Omega} g^{\prime}(s) a(w(t)-w(t-s)) d \Omega d s .
\end{array}
$$

To study the exponential stability of a linear system is of great importance from both the theoretical and practical point of view. First, needless to say, to show the exponential stability of a semigroup has its own merit. Second, it is also important for the study of the global existence of the solution to the corresponding nonlinear system with small initial data (e.g., see [S]). Moreover, as an application, it is known from optimal control theory (see [GRT] and the references cited there) that the exponential stability of a linear system is a sufficient condition for the existence of optimal control for the Linear Quadratic Regulator problem governed by such a system.

In his monograph [L], Lagnese systematically studied thin plate models, including the above thermoelastic and viscoelastic plates, and proved exponential stability when $\Gamma_{1}=\varnothing$ and an appropriate feedback mechanism is implemented along the free edge $\Gamma_{2}$. Since systems (1.2)-(1.3) and (1.12) already possess dissipative mechanisms, an open question is whether they are still exponentially stable without feedback control.

The first result in this direction was obtained by Kim [K] in 1990. He proved the exponential decay of energy of a linear thermoelastic plate $(\gamma=0$ in (1.2)-(1.3)) for rigidly clamped and constant temperature boundary conditions, i.e., on the whole boundary $\Gamma$, 
$w=\frac{\partial w}{\partial \nu}=\theta=0$. More recently, Rivera and Racke $[\mathrm{RR}]$ obtained the same result for the case of $w=\Delta w=\theta=0$ on the whole boundary $\Gamma$. It seems that their methods cannot be applied to the case of more general boundary conditions (1.4)-(1.7).

Our main purpose in this paper is to give a positive answer to the above open problem by showing the exponential stability of semigroups associated with the linear thermoelastic plate equation (1.2)-(1.7) and the linear viscoelastic plate equation (1.12)-(1.16) through a unified treatment. These results are presented in Theorem 2.2 and Theorem 3.2 , respectively. We point out that the results by Kim and Racke can easily be proved by our method. Our method of proof is still a combination of a theorem about the necessary and sufficient conditions for a semigroup to be exponentially stable (see $[\mathrm{H}]$ ) and a contradiction argument. This has been successfully applied to the one-dimensional system with thermal and viscoelastic dampings (see [LZ1], [BLZ], [LZ2]). However, we would like to emphasize that we are now dealing with two-dimensional problems. The information about a positive gap of the eigenvalues of the system, which we heavily used in the proof in our previous papers, is not available. This information is also crucial for the proof of the exponential stability of one-dimensional elastic systems with local damping (see [CFNS]). To overcome this mathematical difficulty, we adopt a different approach without using such information. Therefore, it also throws light on the applications to other higher-dimensional problems.

2. Linear thermoelastic plate. In this section, we first prove the exponential stability of the semigroup associated with the linear thermoelastic plate (1.2)-(1.7), then give a brief discussion to generalize our result to other boundary conditions including the ones treated by Kim and Racke. We set $\gamma=0$ for simplicity. The case of $\gamma \neq 0$ is more delicate and will be treated elsewhere. In what follows, we always assume that $\Gamma_{2} \neq \Gamma$.

Let

$$
Z=H_{\Gamma_{0}}^{2}(\Omega) \cap H_{\Gamma_{1}}^{1}(\Omega) \times L^{2}(\Omega) \times L^{2}(\Omega)
$$

be equipped with the energy-related norm which is induced by the inner product

$$
\langle z, \tilde{z}\rangle_{Z}=a(w, \tilde{w})+\langle v, \tilde{v}\rangle+\beta\langle\theta, \tilde{\theta}\rangle
$$

for any $z=\{w, v, \theta\}, \tilde{z}=\{\tilde{w}, \tilde{v}, \tilde{\theta}\} \in Z$. Hereafter, we denote by $H_{\Gamma_{j}}^{k}(k=1,2, j=0,1)$ the subspace of $H^{k}$ whose elements up to $k-1$ order derivatives vanish on $\Gamma_{j}$ in the trace sense. If we denote $w^{\prime}$ by $v$, then the initial boundary problem (1.2)-(1.7) can be reduced to a first-order evolution equation of the form

$$
z^{\prime}(t)=\mathcal{A} z(t), \quad z(0)=z_{0}
$$

where $z_{0}=\left\{w^{0}, w^{1}, \theta^{0}\right\}$ and

$$
\begin{gathered}
\mathcal{A}=\left[\begin{array}{ccc}
0 & I & 0 \\
-\Delta^{2} & 0 & -\alpha \Delta \\
0 & \frac{\alpha}{\beta} \Delta & \frac{1}{\beta}(-\sigma I+\eta \Delta)
\end{array}\right], \\
\mathcal{D}(\mathcal{A})=\left\{\begin{array}{ll}
\left.z \in Z: \begin{array}{l}
w \in H^{4}(\Omega) \cap H_{\Gamma_{0}}^{2}(\Omega) \cap H_{\Gamma_{1}}^{1}(\Omega), v \in H_{\Gamma_{0}}^{2}(\Omega) \cap H_{\Gamma_{1}}^{1}(\Omega), \\
\theta \in H^{2}(\Omega),
\end{array}\right\}, \theta \text { satisfy }(1.4)-(1.7)
\end{array}\right\} .
\end{gathered}
$$


We recall the following Green's formula (see $[\mathrm{L}]$ ) since it will be used extensively in the rest of this paper:

$$
\int_{\Omega}\left(\Delta^{2} w\right) \tilde{w} d \Omega=a(w ; \tilde{w})+\int_{\Gamma}\left\{\left[\frac{\partial \Delta w}{\partial \nu}+(1-\mu) \frac{\partial B_{2} w}{\partial \tau}\right] \tilde{w}-\left[\Delta w+(1-\mu) B_{1} w\right] \frac{\partial \tilde{w}}{\partial \nu}\right\} d \Gamma
$$

Theorem 2.1. The operator $\mathcal{A}$ defined by (2.4)-(2.5) is the infinitesimal generator of a $C_{0}$-semigroup, $T(t)$, of contractions on $H_{\Gamma_{0}}^{2}(\Omega) \cap H_{\Gamma_{1}}^{1}(\Omega) \times L^{2}(\Omega) \times L^{2}(\Omega)$.

Proof. It is clear that $\mathcal{D}(\mathcal{A})$ is dense in $Z$. For any $z=\{w, v, \theta\} \in \mathcal{D}(\mathcal{A})$,

$$
\begin{aligned}
\operatorname{Re}\langle\mathcal{A} z, z\rangle_{Z}= & \operatorname{Re}\left\{a(v, w)+\left\langle-\Delta^{2} w-\alpha \Delta \theta, v\right\rangle+\langle\alpha \Delta v-\sigma \theta+\eta \Delta \theta, \theta\rangle\right\} \\
= & \operatorname{Re}\left\{a(v, w)-a(w, v)-\int_{\Gamma}\left\{\left[\frac{\partial \Delta w}{\partial \nu}+(1-\mu) \frac{\partial B_{2} w}{\partial \tau}-\alpha \frac{\partial \theta}{\partial \nu}\right] v\right.\right. \\
& \left.\left.-\left[\Delta w+(1-\mu) B_{1} w-\alpha \theta\right] \frac{\partial v}{\partial \nu}\right\} d \Gamma-\alpha\langle\theta, \Delta v\rangle+\langle\alpha \Delta v-\sigma \theta+\eta \Delta \theta, \theta\rangle\right\} \\
= & -\sigma\|\theta\|^{2}+\eta\langle\Delta \theta, \theta\rangle \\
= & -\sigma\|\theta\|^{2}-\eta\|\nabla \theta\|^{2}-\int_{\Gamma^{2}} \theta^{2} d \Gamma \leq 0
\end{aligned}
$$

where we have used Green's formula (2.6) and the boundary conditions (1.4)-(1.7). Hereafter, we denote by $\|\cdot\|$ the usual $L^{2}(\Omega)$ norm. Thus $\mathcal{A}$ is dissipative. It remains to show that Range $(I-\mathcal{A})=Z$, i.e., for any $F=\left\{f_{1}, f_{2}, f_{3}\right\} \in Z$, we want to show that the equation

$$
(I-\mathcal{A}) z=F
$$

has a unique solution $z$. Instead of (2.8), we look for $w \in H^{4}$ and $\theta \in H^{2}$ satisfying

$$
\begin{aligned}
w+\Delta^{2} w+\alpha \Delta \theta & =f_{1}+f_{2}, \\
\beta \theta-\alpha \Delta w+\sigma \theta-\eta \Delta \theta & =f_{3}-\alpha \Delta f_{1}
\end{aligned}
$$

and the boundary conditions (1.4)-(1.7).

Let $y=\{w, \theta\}$. We associate this problem with the following bilinear form on $H_{\Gamma_{0}}^{2} \cap$ $H_{\Gamma_{1}}^{1} \times H^{1}$ :

$$
\begin{aligned}
b(y, \tilde{y})= & \int_{\Omega} w \tilde{w} d \Omega+a(w, \tilde{w})+\alpha \int_{\Omega}(\theta \Delta \tilde{w}-\tilde{\theta} \Delta w) d \Omega \\
& +(\beta+\sigma) \int_{\Omega} \theta \tilde{\theta} d \Omega+\eta \int_{\Omega} \nabla \theta \nabla \tilde{\theta} d \Omega+\eta \lambda \int_{\Gamma} \theta \tilde{\theta} d \Gamma
\end{aligned}
$$

Then by the well-known Lax-Milgram theorem, there is a unique solution $y \in H_{\Gamma_{0}}^{2} \cap$ $H_{\Gamma_{1}}^{1} \times H^{1}$ such that

$$
b(y, \tilde{y})=\int_{\Omega}\left[\left(f_{1}+f_{2}\right) \tilde{w}+\left(f_{3}-\alpha \Delta f_{1}\right) \tilde{\theta}\right] d \Omega, \quad \forall \tilde{y} \in H_{\Gamma_{0}}^{2} \cap H_{\Gamma_{1}}^{1} \times H^{1} .
$$


This implies that $\theta \in H^{1}$ is a weak solution of the following elliptic boundary-value problem:

$$
\begin{aligned}
(\beta+\sigma) \theta-\eta \Delta \theta & =f_{3}-\alpha \Delta f_{1}+\alpha \Delta w \in L^{2}(\Omega) \\
\left.\left(\frac{\partial \theta}{\partial \nu}+\lambda \theta\right)\right|_{\Gamma} & =0 .
\end{aligned}
$$

By the regularity theorem (see $[\mathrm{LM}]), \theta \in H^{2}$.

On the other hand, (2.12) also implies that $w \in H_{\Gamma_{0}}^{2} \cap H_{\Gamma_{1}}^{1}$ is a weak solution of the following elliptic boundary-value problem:

$$
\begin{aligned}
& w+\Delta^{2} w=f_{1}+f_{2}-\alpha \Delta \theta \in L^{2}, \quad \text { in } \Omega, \\
& w=\frac{\partial w}{\partial \nu}=0, \quad \text { on } \Gamma_{0}, \\
& w=0, \quad \Delta w+(1-\mu) B_{1} w=-\alpha \theta \in H^{\frac{3}{2}}\left(\Gamma_{1}\right), \quad \text { on } \Gamma_{1}, \\
& \left\{\begin{array}{l}
\Delta w+(1-\mu) B_{1} w=-\alpha \theta \in H^{\frac{3}{2}}\left(\Gamma_{2}\right), \\
\frac{\partial \Delta w}{\partial \nu}+(1-\mu) \frac{\partial B_{2} w}{\partial \nu}=-\alpha \frac{\partial \theta}{\partial \nu} \in H^{\frac{1}{2}}\left(\Gamma_{2}\right),
\end{array} \quad \text { on } \Gamma_{2} .\right.
\end{aligned}
$$

Thus by the regularity theorem again, we have

$$
w \in H^{4} \cap H_{\Gamma_{0}}^{2} \cap H_{\Gamma_{1}}^{1}
$$

Let

$$
v=w-f_{1} \in H_{\Gamma_{0}}^{2} \cap H_{\Gamma_{1}}^{1} .
$$

Combining (2.20) with (2.13)-(2.19) yields that $z=\{w, v, \theta\}$ belongs to $\mathcal{D}(\mathcal{A})$ and satisfies (2.8).

Theorem 2.2. Suppose $\Gamma_{2}=\varnothing$. Then the semigroup $T(t)$ in Theorem 2.1 is exponentially stable, i.e., there exist $M, \delta>0$ such that

$$
\|T(t)\|_{\mathcal{L}(Z, Z)} \leq M e^{-\delta t}, \quad t>0 .
$$

Proof. By a result of Huang $[\mathrm{H}]$, the exponential stability of $T(t)$ is equivalent to

$$
\sup \{\operatorname{Re} \lambda: \lambda \in \text { spectrum of } \mathcal{A}\}<0
$$

and

$$
\sup \left\{\left\|(\lambda I-A)^{-1}\right\|_{L(Z, Z)}: \operatorname{Re} \lambda \geq 0\right\}=K<\infty .
$$

Hence if the conclusion in Theorem 2.2 is false, then one of (2.22) and (2.23) must fail to hold. Assume that (2.23) fails. There must exist a sequence of $\lambda_{n} \in \mathbf{C}$ and a sequence of $z_{n}=\left\{w_{n}, v_{n}, \theta_{n}\right\} \in \mathcal{D}(\mathcal{A})$ with $\operatorname{Re} \lambda_{n} \geq 0,\left\|z_{n}\right\|_{Z}=1$ such that

$$
\left(\lambda_{n} I-\mathcal{A}\right) z_{n} \rightarrow 0 \quad \text { in } Z
$$


or

$$
\begin{aligned}
a\left(\lambda_{n} w_{n}-v_{n}\right) & \rightarrow 0, \\
\lambda_{n} v_{n}+\Delta^{2} w_{n}+\alpha \Delta \theta_{n} & \rightarrow 0 \quad \text { in } L^{2}(\Omega), \\
\lambda_{n} \beta \theta_{n}-\alpha \Delta v_{n}+\sigma \theta_{n}-\eta \Delta \theta_{n} \rightarrow 0 & \text { in } L^{2}(\Omega) .
\end{aligned}
$$

By (2.7), we have

$$
\operatorname{Re}\left\langle\left(\lambda_{n} I-\mathcal{A}\right) z_{n}, z_{n}\right\rangle_{Z}=\operatorname{Re} \lambda_{n}+\sigma\left\|\theta_{n}\right\|^{2}+\eta\left\|\nabla \theta_{n}\right\|^{2}+\eta \lambda \int_{\Gamma} \theta_{n}^{2} d \Gamma
$$

Since each term on the right side of (2.28) is nonnegative, it follows from (2.24) that

$$
\operatorname{Re} \lambda_{n} \rightarrow 0, \quad \theta_{n} \rightarrow 0 \quad \text { in } H^{1}(\Omega)
$$

which further leads to

$$
a\left(w_{n}\right)+\|v\|^{2} \rightarrow 1
$$

It is easy to see (e.g., $[\operatorname{Re}])$ that

$$
a(w) \geq c\|w\|_{H^{2}}^{2}, \quad \forall w \in H_{\Gamma_{0}}^{2}(\Omega) \cap H_{\Gamma_{1}}^{1}(\Omega)
$$

for some constant $c>0$. Therefore (2.25) implies

$$
\lambda_{n} w_{n}-v_{n} \rightarrow 0 \text { in } L^{2}(\Omega) .
$$

Taking the complex conjugate of the inner product of (2.32) with $v_{n}$ in $L^{2}(\Omega)$, then adding it to the inner product of $(2.26)$ with $w_{n}$ in $L^{2}(\Omega)$, we obtain

$$
2 \operatorname{Re} \lambda_{n} \cdot\langle v, w\rangle+a\left(w_{n}\right)-\left\|v_{n}\right\|^{2}+\alpha\left\langle\theta_{n}, \Delta w_{n}\right\rangle \rightarrow 0
$$

It follows from (2.29) that the first term in (2.33) goes to zero. The last inner product in (2.33) also converges to zero because $\left\|\Delta w_{n}\right\| \leq 1$ and $\left\|\theta_{n}\right\| \rightarrow 0$. Combining (2.33) with (2.30) yields

$$
a\left(w_{n}\right) \rightarrow \frac{1}{2}, \quad\left\|v_{n}\right\|^{2} \rightarrow \frac{1}{2}
$$

In the rest of the proof, we shall show (2.34) is a contradiction. We first claim that $\left|\lambda_{n}\right| \geq \varepsilon>0$ for all $n$ large enough. Otherwise, there exists a subsequence of $\lambda_{n}$, still denoted by $\lambda_{n}$, which converges to zero. We obtain from (2.25) that

$$
a\left(v_{n}\right) \rightarrow 0
$$

Thus $v_{n}$ must converge to zero in $L^{2}(\Omega)$, which contradicts (2.34).

Now we divide (2.27) by $\lambda_{n}$ and apply (2.25) and (2.29) to get

$$
\alpha \Delta w_{n}+\frac{\eta}{\lambda_{n}} \Delta \theta_{n} \rightarrow 0 \quad \text { in } L^{2}(\Omega)
$$


It is easy to see from (2.36) that the term $\left\|\frac{\Delta \theta_{n}}{\lambda_{n}}\right\|$ is bounded uniformly in $n$. Thus combining this with (2.26) yields the uniform boundedness of $\left\|\frac{\Delta^{2} w_{n}}{\lambda_{n}}\right\|$ in $n$. Taking the inner product of $(2.36)$ with $\Delta w_{n}$ in $L^{2}(\Omega)$ yields

$$
\begin{aligned}
& \alpha\left\|\Delta w_{n}\right\|^{2}+\eta\left\langle\frac{\Delta \theta_{n}}{\lambda_{n}}, \Delta w_{n}\right\rangle \\
& \quad=\alpha\left\|\Delta w_{n}\right\|^{2}+\eta \int_{\Gamma} \frac{\Delta w_{n}}{\lambda_{n}} \frac{\partial \theta_{n}}{\partial \nu} d \Gamma-\eta \int_{\Gamma} \frac{\theta_{n}}{\lambda_{n}} \frac{\partial\left(\Delta w_{n}\right)}{\partial \nu} d \Gamma+\eta\left\langle\theta_{n}, \frac{\Delta^{2} w_{n}}{\lambda_{n}}\right\rangle \\
& \quad \rightarrow 0 .
\end{aligned}
$$

Now it follows from (2.29) that the last inner product of (2.37) must converge to zero. We estimate the two boundary integrals in (2.37) as follows. By the trace theorem, we have

$$
\begin{aligned}
\left|\int_{\Gamma} \frac{\Delta w_{n}}{\lambda_{n}} \frac{\partial \theta_{n}}{\partial \nu} d \Gamma\right| & =\left|\lambda \int_{\Gamma} \frac{\Delta w_{n}}{\lambda_{n}} \theta_{n} d \Gamma\right| \\
& \leq \lambda\left\|\frac{\Delta w_{n}}{\lambda_{n}}\right\|_{L^{2}(\Gamma)} \cdot\left\|\theta_{n}\right\|_{L^{2}(\Gamma)} \\
& \leq C \cdot\left\|\frac{\Delta w_{n}}{\lambda_{n}}\right\|_{H^{1}(\Omega)} \cdot\left\|\theta_{n}\right\|_{H^{1}(\Omega)} .
\end{aligned}
$$

Hereafter, $C$ is a positive constant which may vary in different places. Notice that $w_{n}$ satisfies the boundary conditions (1.4)-(1.6). Thus the standard estimates for the elliptic boundary-value problem and the trace theorem (see [LM]) lead to

$$
\begin{aligned}
\left\|w_{n}\right\|_{H^{4}(\Omega)} & \leq C\left(\left\|\Delta^{2} w_{n}\right\|+\left\|\alpha \theta_{n}\right\|_{H^{\frac{3}{2}(\Gamma)}}\right) \\
& \leq C\left(\left\|\Delta^{2} w_{n}\right\|+\left\|\theta_{n}\right\|_{H^{2}(\Omega)}\right) \\
& \leq C\left(\left\|\Delta^{2} w_{n}\right\|+\left\|\Delta \theta_{n}\right\|+\left\|\theta_{n}\right\|_{H^{1}(\Omega)}\right), \\
\left\|\frac{w_{n}}{\lambda_{n}}\right\|_{H^{4}(\Omega)} & \leq C\left(\left\|\frac{\Delta^{2} w_{n}}{\lambda_{n}}\right\|+\left\|\frac{\Delta \theta_{n}}{\lambda_{n}}\right\|+\left\|\frac{\theta_{n}}{\lambda_{n}}\right\|_{H^{1}(\Omega)}\right) .
\end{aligned}
$$

Thus it follows from (2.38), (2.40), and (2.29) that

$$
\left|\int_{\Gamma} \frac{\Delta w_{n}}{\lambda_{n}} \frac{\partial \theta_{n}}{\partial \nu} d \Gamma\right| \rightarrow 0
$$

Similarly, we have

$$
\left|\int_{\Gamma} \frac{\theta_{n}}{\lambda_{n}} \frac{\partial\left(\Delta w_{n}\right)}{\partial \nu} d \Gamma\right| \rightarrow 0 .
$$

It turns out from (2.37) that

$$
\Delta w_{n} \rightarrow 0 \text { in } L^{2}(\Omega) .
$$

Since $\Gamma_{2}=\varnothing, w_{n}=0$ on the whole boundary $\Gamma$. By the estimates of the elliptic boundary-value problem, we have

$$
a\left(w_{n}\right) \leq C \cdot\left\|w_{n}\right\|_{H^{2}(\Omega)}^{2} \leq C \cdot\left\|\Delta w_{n}\right\|^{2} \rightarrow 0 .
$$


This contradicts (2.34).

Now we prove that (2.22) also holds. Because $T(t)$ is a $C_{0}$ semigroup of contractions, $\rho(\mathcal{A})$ contains the set $\{\lambda \mid \operatorname{Re} \lambda>0\}$. For any $\sigma \in\left[0, \frac{1}{2 K}\right]$ and $\omega \in \mathbf{R}$, we have

$$
(-\sigma+i \omega-\mathcal{A})^{-1}=\left(\frac{1}{4 K}+i \omega-\mathcal{A}\right)^{-1}\left[1-\left(\sigma+\frac{1}{4 K}\right)\left(\frac{1}{4 K}+i \omega-\mathcal{A}\right)^{-1}\right]^{-1}
$$

Thus,

$$
\sup \{\operatorname{Re} \lambda ; \lambda \in \sigma(\mathcal{A})\}<-\frac{1}{4 K} .
$$

Thus the proof of Theorem 2.2 is completed.

REMARK 2.1. It is easy to see that if $w=\Delta w=\theta=0$ on the whole boundary $\Gamma$, as considered by Rivera and Racke [RR], the conclusion of Theorem 2.2 remains true without any modifications of the proof.

If the boundary conditions are $w=\frac{\partial w}{\partial \nu}=\theta=0$ on the whole boundary $\Gamma$, as considered by $\operatorname{Kim}[\mathrm{K}]$, then we modify our proof as follows.

The proof until (2.36) still works. From (2.36), we have

$$
\left\|\alpha w_{n}+\frac{\theta_{n}}{\lambda_{n}}\right\|_{H^{2}(\Omega)} \leq C\left\|\Delta\left(\alpha w_{n}+\frac{\theta_{n}}{\lambda_{n}}\right)\right\| \rightarrow 0 .
$$

Combining it with $(2.29)$ yields

$$
w_{n} \rightarrow 0 \text { in } H^{1}(\Omega) .
$$

We divide $(2.26)$ by $\lambda_{n}$ and then take the inner product with $-\Delta w_{n}$ in $L^{2}(\Omega)$ to obtain

$$
\left\langle v_{n},-\Delta w_{n}\right\rangle-\frac{1}{\lambda_{n}}\left\langle\Delta^{2} w_{n}, \Delta w_{n}\right\rangle+\frac{\alpha^{2}}{\eta}\left\|\Delta w_{n}\right\|^{2} \rightarrow 0 .
$$

Here we have also used (2.36).

Taking (2.32) into consideration and noticing that

$$
-\left\langle\Delta^{2} w_{n}, \Delta w_{n}\right\rangle=-\int_{\Gamma} \frac{\partial \Delta w_{n}}{\partial \nu} \cdot \overline{\Delta w_{n}} d \Gamma+\left\|\nabla\left(\Delta w_{n}\right)\right\|^{2}
$$

is a real number, we obtain

$$
\operatorname{Re} \lambda_{n} \cdot\left\|\nabla w_{n}\right\|^{2}+\frac{\operatorname{Re} \lambda_{n}}{\left|\lambda_{n}\right|^{2}}\left(\left\|\nabla\left(\Delta w_{n}\right)\right\|^{2}-\int_{\Gamma} \frac{\partial \Delta w_{n}}{\partial \nu} \overline{\Delta w_{n}} d \Gamma\right)+\frac{\alpha^{2}}{\eta}\left\|\Delta w_{n}\right\|^{2} \rightarrow 0 .
$$

Owing to (2.29) and (2.46), the first term on the left-hand side of (2.49) converges to zero. Since $\left\|\frac{\Delta^{2} w_{n}}{\lambda_{n}}\right\|$ is bounded, by the estimates of elliptic boundary-value problems and the trace theorem,

$$
\frac{1}{\left|\lambda_{n}\right|^{2}}\left(\left\|\nabla\left(\Delta w_{n}\right)\right\|^{2}-\int_{\Gamma} \frac{\partial \Delta w_{n}}{\partial \nu} \overline{\Delta w_{n}} d \Gamma\right)
$$

is also bounded. Therefore, the second term in (2.49) also converges to zero. It turns out that

$$
a\left(w_{n}\right) \leq C \cdot\left\|w_{n}\right\|_{H^{2}(\Omega)}^{2} \leq C \cdot\left\|\Delta w_{n}\right\|^{2} \rightarrow 0,
$$

a contradiction. Thus the result by Kim $[\mathrm{K}]$ can also be proved by our method.

REMARK 2.2. It is still an open question whether the exponential stability remains true if $\Gamma_{2} \neq \varnothing$. 
3. Linear viscoelastic plate. In this section, we turn to the exponential stability of the semigroup associated with the linear viscoelastic plate (1.12)-(1.15). We choose the function spaces

$$
Z=H_{\Gamma_{0}}^{2}(\Omega) \cap H_{\Gamma_{1}}^{1}(\Omega) \times L^{2}(\Omega) \times L^{2}\left(0, \infty ;\left|g^{\prime}(\cdot)\right| ; H_{\Gamma_{0}}^{2}(\Omega) \cap H_{\Gamma_{1}}^{1}(\Omega)\right)
$$

equipped with the norm

$$
\|\{w, v, h\}\|_{Z}=\left(g_{\infty} a(w)+\|v\|^{2}+\int_{0}^{\infty}\left|g^{\prime}(s)\right| a(h) d s\right)^{1 / 2} .
$$

If we introduce the variables

$$
v=w^{\prime}, \quad h=w(t)-w(t-s),
$$

then the space $Z$ is a "finite energy space" of the type first introduced for viscoelastic problems by Dafermos ([D1], [D2]), where the norm square of the state variables is exactly twice that of the system energy defined in (1.18).

System (1.12)-(1.15) corresponds to the abstract evolution equation

$$
z^{\prime}=\mathcal{A} z, \quad z(0)=z_{0} .
$$

Here, $z=\{w, v, h\}, z_{0}=\left\{w^{0}, w^{1}, w_{h}\right\}$ and

$$
\mathcal{A} z=\left(\begin{array}{c}
v \\
-g_{\infty} \Delta^{2} w+\int_{0}^{\infty} g^{\prime}(s) \Delta^{2} h d s \\
v-\frac{\partial}{\partial s} h
\end{array}\right),
$$

with

$$
\mathcal{D}(\mathcal{A})=\left\{z \in Z|\mathcal{A} z \in Z, h|_{s=0}=0, w \text { satisfies (1.13)-(1.15) }\right\} .
$$

Theorem 3.1. The operator $\mathcal{A}$ defined by (3.5)-(3.6) is an infinitesimal generator of a $C_{0}$-semigroup $T(t)$ of contractions on $H_{\Gamma_{0}}^{2}(\Omega) \cap H_{\Gamma_{1}}^{1}(\Omega) \times L^{2}(\Omega) \times L^{2}\left(0, \infty ;\left|g^{\prime}(\cdot)\right| ; H_{\Gamma_{0}}^{2}(\Omega) \cap\right.$ $\left.H_{\Gamma_{1}}^{1}(\Omega)\right)$.

Proof. Actually, the fact that $T(t)$ is a $C_{0}$-semigroup has already been proved in [FI] in an abstract setting. Moreover, as in [LZ2], for any $z=\{w, v, h\} \in \mathcal{D}(\mathcal{A})$,

$$
\begin{aligned}
\operatorname{Re}\langle\mathcal{A} z, z\rangle_{Z}= & \operatorname{Re}\left\{g_{\infty} a(v, w)-\left\langle g_{\infty} \Delta^{2} w+\int_{0}^{\infty} g^{\prime}(s) \Delta^{2} h(s), v\right\rangle\right. \\
& \left.-\int_{0}^{\infty} g^{\prime}(s) a\left(v-\frac{\partial h}{\partial s}, h\right) d s\right\} \\
= & \operatorname{Re}\left\{-\int_{\Gamma}\left[\mathcal{B}_{1}\left(w(t)+\int_{0}^{\infty} g^{\prime}(s) w(t-s) d s\right)\right] \frac{\partial v}{\partial \nu} d \Gamma\right. \\
& \left.+\int_{\Gamma}\left[\mathcal{B}_{2}\left(w(t)+\int_{0}^{\infty} g^{\prime}(s) w(t-s) d s\right)\right] v d \Gamma+\int_{0}^{\infty} g^{\prime}(s) a\left(\frac{\partial h}{\partial s}, h\right) d s\right\} \\
= & -\frac{1}{2} \int_{0}^{\infty} g^{\prime \prime}(s) a(h) d s \leq 0 .
\end{aligned}
$$


We also refer to $[\mathrm{L}]$ for a slightly different framework. Lagnese [L] obtained exponential stability for a slightly restrictive kernel $g$ by imposing an appropriate feedback boundary control on $\Gamma_{2}$ and assuming $\Gamma_{1}=\varnothing$. He also conjectured that various asymptotic decay rates for the solution of (1.12) may be obtained without recourse to boundary feedback, i.e., based only on the properties of the kernel $g(s)$. Our following theorem gives a positive answer to his conjecture.

Theorem 3.2. Suppose $\Gamma_{2} \neq \Gamma$. Then the $C_{0}$-semigroup $T(t)$ in Theorem 3.1 is exponentially stable, i.e., there exist constant $M ; \delta>0$ such that

$$
\|T(t)\|_{\mathcal{L}(Z, Z)} \leq M e^{-\delta t}, \quad t>0 .
$$

Proof. Suppose (2.23) is not true. Then, there exists a sequence $\lambda_{n} \in \mathbf{C}$ and a sequence of unit vectors $z_{n}=\left\{w_{n}, v_{n}, h_{n}\right\} \in \mathcal{D}(\mathcal{A})$ with $\operatorname{Re} \lambda_{n} \geq 0$ and $\left\|z_{n}\right\|_{Z}=1$ such that

$$
\left(\lambda_{n} I-\mathcal{A}\right) z_{n} \rightarrow 0 \quad \text { in } Z \text { as } n \rightarrow \infty
$$

This is equivalent to

$$
\begin{aligned}
a\left(\lambda_{n} w_{n}-v_{n}\right) & \rightarrow 0 \\
\lambda_{n} v_{n}+g_{\infty} \Delta^{2} w_{n}+\int_{0}^{\infty} g^{\prime}(s) \Delta^{2} h_{n} d s & \rightarrow 0 \quad \text { in } L^{2}(\Omega) \\
\lambda_{n} h_{n}-v_{n}+\frac{\partial}{\partial s} h_{n} & \rightarrow 0 \quad \text { in } L^{2}\left(0, \infty ;\left|g^{\prime}(s)\right| ; H_{\Gamma_{0}}^{2}(\Omega) \cap H_{\Gamma_{1}}^{1}(\Omega)\right) .
\end{aligned}
$$

On the other hand, by (g4) and (3.9), we have

$$
\begin{aligned}
-\frac{k}{2} \int_{0}^{\infty} g^{\prime}(s) a\left(h_{n}\right) d s & \leq \operatorname{Re} \lambda_{n}+\frac{1}{2} \int_{0}^{\infty} g^{\prime \prime}(s) a\left(h_{n}\right) d s \\
& =\operatorname{Re}\left\langle\left(\lambda_{n} I-\mathcal{A}\right) z_{n}, z_{n}\right\rangle_{Z} \\
& \leq\left\|\left(\lambda_{n} I-\mathcal{A}\right) z_{n}\right\|_{Z} \rightarrow 0 .
\end{aligned}
$$

Therefore,

$$
\begin{aligned}
& \int_{0}^{\infty} g^{\prime}(s) a\left(h_{n}\right) d s \rightarrow 0, \\
\operatorname{Re} \lambda_{n} \rightarrow 0, \quad & \int_{0}^{\infty} g^{\prime \prime}(s) a\left(h_{n}\right) d s \rightarrow 0,
\end{aligned}
$$

which implies that

$$
g_{\infty} a\left(w_{n}\right)+\left\|v_{n}\right\|^{2} \rightarrow 1 .
$$

Since $\Gamma_{2} \neq \Gamma$, it follows from (3.10) that

$$
\lambda_{n} w_{n}-v_{n} \rightarrow 0 \text { in } H^{2}(\Omega)
$$


Taking the inner product of (3.17) with $v_{n}$ and (3.11) with $w_{n}$ in $L^{2}(\Omega)$, and applying Green's formula and the boundary conditions, we obtain

$$
\begin{aligned}
\lambda_{n}\left\langle w_{n}, v_{n}\right\rangle-\left\|v_{n}\right\|^{2} & \rightarrow 0, \\
\lambda_{n}\left\langle v_{n}, w_{n}\right\rangle+g_{\infty} a\left(w_{n}\right)+\int_{0}^{\infty} g^{\prime}(s) a\left(h_{n}, w_{n}\right) d s & \rightarrow 0 .
\end{aligned}
$$

The last term in (3.19) converges to zero since

$$
\begin{aligned}
& \left|\int_{0}^{\infty} g^{\prime}(s) a\left(h_{n}, w_{n}\right) d s\right| \\
& \quad \leq\left[a\left(w_{n}\right)\right]^{1 / 2} \int_{0}^{\infty}-g^{\prime}(s)\left[a\left(h_{n}\right)\right]^{1 / 2} d s \\
& \quad \leq\left(1-g_{\infty}\right) \int_{0}^{\infty}-g^{\prime}(s) a\left(h_{n}\right) d s \rightarrow 0 .
\end{aligned}
$$

By adding the complex conjugate of (3.18) to (3.19), we obtain

$$
\operatorname{Re} \lambda_{n} \cdot\left\langle v_{n}, w_{n}\right\rangle+g_{\infty} a\left(w_{n}\right)-\left\|v_{n}\right\|^{2} \rightarrow 0
$$

Now (3.16) and (3.21) imply that

$$
g_{\infty} a\left(w_{n}\right) \rightarrow \frac{1}{2}, \quad\left\|v_{n}\right\|^{2} \rightarrow \frac{1}{2}
$$

In what follows, we shall show that $(3.22)$ is a contradiction. We first claim that $\left|\lambda_{n}\right| \geq \varepsilon>0$ for $n$ large enough. Otherwise, there exists a subsequence, denoted by $\lambda_{n}$ again for simplicity, such that $\lambda_{n} \rightarrow 0$. By (3.17), $v_{n}$ must converge to zero in $L^{2}(\Omega)$. Contradiction.

Next, we divide (3.12) by $\lambda_{n}$, then take the inner product with $\frac{s v_{n}}{\lambda_{n}}$ in $L^{2}\left(0, \infty ;\left|g^{\prime}(s)\right|\right.$; $\left.H_{\Gamma_{0}}^{2} \cap H_{\Gamma_{1}}^{1}(\Omega)\right)$ to obtain

$$
\int_{0}^{\infty} s g^{\prime}(s) a\left(h_{n}, \frac{v_{n}}{\lambda_{n}}\right) d s-a\left(\frac{v_{n}}{\lambda_{n}}\right) \int_{0}^{\infty} s g^{\prime}(s) d s+\frac{1}{\lambda_{n}} \int_{0}^{\infty} s g^{\prime}(s) a\left(\frac{\partial h_{n}}{\partial s}, \frac{v_{n}}{\lambda_{n}}\right) d s \rightarrow 0 .
$$

The first and third term in (3.23) can be estimated as follows:

$$
\begin{aligned}
\left|\int_{0}^{\infty} s g^{\prime}(s) a\left(h_{n}, \frac{v_{n}}{\lambda_{n}}\right) d s\right| & \leq\left[a\left(\frac{v_{n}}{\lambda_{n}}\right)\right]^{\frac{1}{2}} \int_{0}^{\infty}-s g^{\prime}(s)\left[a\left(h_{n}\right)\right]^{\frac{1}{2}} d s \\
& \leq\left[a\left(\frac{v_{n}}{\lambda_{n}}\right)\right]^{\frac{1}{2}}\left(\int_{0}^{\infty}-s^{2} g^{\prime}(s) d s\right)^{\frac{1}{2}}\left(\int_{0}^{\infty}-g^{\prime}(s) a\left(h_{n}\right) d s\right)^{\frac{1}{2}} \\
& \leq C \cdot\left(\int_{0}^{\infty}-g^{\prime}(s) a\left(h_{n}\right) d s\right)^{1 / 2} \rightarrow 0
\end{aligned}
$$


and

$$
\begin{gathered}
\left|\frac{1}{\lambda_{n}} \int_{0}^{\infty}-s g^{\prime}(s) a\left(\frac{\partial h_{n}}{\partial s}, \frac{v_{n}}{\lambda_{n}}\right) d s\right| \leq \frac{1}{\varepsilon}\left|\int_{0}^{\infty}\left(s g^{\prime \prime}(s)+g^{\prime}(s)\right) a\left(h_{n}, \frac{v_{n}}{\lambda_{n}}\right) d s\right| \\
\leq \frac{1}{\varepsilon}\left[a\left(\frac{v_{n}}{\lambda_{n}}\right)\right]^{\frac{1}{2}}\left[\left(\int_{0}^{\infty} s^{2} g^{\prime \prime}(s) d s\right)^{1 / 2}\left(\int_{0}^{\infty} g^{\prime \prime}(s) a\left(h_{n}\right) d s\right)^{1 / 2}\right. \\
\left.+\left(\int_{0}^{\infty}-g^{\prime}(s) d s\right)^{1 / 2}\left(\int_{0}^{\infty}-g^{\prime}(s) a\left(h_{n}\right) d s\right)^{1 / 2}\right] \\
\leq C \cdot\left[\left(\int_{0}^{\infty} g^{\prime \prime}(s) a\left(h_{n}\right) d s\right)^{1 / 2}+\left(\int_{0}^{\infty}-g^{\prime}(s) a\left(h_{n}\right) d s\right)^{1 / 2}\right] \rightarrow 0 .
\end{gathered}
$$

Here we have used uniform boundedness of $a\left(\frac{v_{n}}{\lambda_{n}}\right)$ in $n$ by (3.17) and the condition on $g(s)$ as well as the convergence results in (3.14)-(3.15). Thus the second term in (3.23) must also converge to zero, which is equivalent to

$$
a\left(\frac{v_{n}}{\lambda_{n}}\right) \rightarrow 0
$$

Finally, by (3.17) again, we have

$$
a\left(w_{n}\right) \rightarrow 0 .
$$

This is a contradiction. Condition (2.22) can be verified by the same argument as in the proof of Theorem 2.2.

\section{REFERENCES}

[BLZ] J. A. Burns, Z. Liu, and S. Zheng, On the energy decay of a linear thermoelastic bar, J. Math. Anal. Appl. 179, 574-591 (1993)

[CFNS] G. Chen, S. A. Fulling, F. J. Narcowich, and S. Sun, Exponential decay of evolution equations with locally distributed damping, SIAM J. Appl. Math. 51, 266-301 (1991)

[D1] C. M. Dafermos, Asymptotic stability in viscoelasticity, Arch. Rat. Mech. Anal. 37, 297-308 (1970)

[D2] C. M. Dafermos, An abstract Volterra equation with applications to linear viscoelasticity, J. Differential Equations 7, 554-569 (1970)

[DM] W. Desch and R. K. Miller, Exponential stabilization of Volterra integrodifferential equations in Hilbert space, J. Differential Equations 70, 366-389 (1987)

[FI] R. H. Fabiano and K. Ito, Semigroup theory and numerical approximation for equations arising in linear viscoelasticity, SIAM J. Math. Anal. 21, 374-393 (1990)

[GRT] J. S. Gibson, I. G. Rosen, and G. Tao, Approximation in control of thermoelastic systems, SIAM J. Control. Optim. 30, 1163-1189 (1992)

[H] F. L. Huang, Characteristic condition for exponential stability of linear dynamical system in Hilbert spaces, Ann. Differential Equations 1, 43-56 (1985)

[HRW] K. B. Hannsgen, Y. Renardy, and R. L. Wheeler, Effectiveness and robustness with respect to time delays of boundary feedback stabilization in one-dimensional viscoelasticity, SIAM J. Control Optim. 26, 1200-1233 (1988)

[K] J. U. Kim, On the energy decay of a linear thermoelastic bar and plate, SIAM J. Math. Anal. 23, 889-899 (1992)

[L] J. Lagnese, Boundary Stabilization of Thin Plates, Vol. 10 of SIAM Studies in Applied Mathematics, Society for Industrial and Applied Mathematics, Philadelphia, 1989

[La] I. Lasiecka, Controllability of a viscoelastic Kirchhoff plate, Internat. Ser. Numer. Math. 91, $237-247$ (1989) 
[LM] J. L. Lions and E. Magenes, Nonhomogeneous Boundary Value Problems and Applications, Springer, Heidelberg, 1972

[LZ1] Z. Liu and S. Zheng, Exponential stability of semigroup associated with thermoelastic system, Quart. Appl. Math. LI, 535-545 (1993)

[LZ2] Z. Liu and S. Zheng, On the exponential stability of linear viscoelasticity and thermoviscoelasticity, Quart. Appl. Math. LIV, 21-31 (1996)

[Pa] A. Pazy, Semigroups of Linear Operators and Applications to Partial Differential Equations, Springer, New York, 1983

[RR] J. E. M. Rivera and R. Racke, Smoothing properties, decay and global existence of solutions to nonlinear coupled systems of thermoelastic type, SFB series No. 287, Bonn University, 1993

[Re] K. Rektorys, Variational Methods in Mathematics, Science and Engineering, D. Reidel Publishing Company, Holland, 1977

[S] M. Slemrod, Global existence, uniqueness, and asymptotic stability of classical smooth solutions in one-dimensional nonlinear thermoelasticity, Arch. Rational Mech. Anal. 76, 97-133 (1981) 